\title{
Review: early mobilisation is better than bed rest for medical conditions and after healthcare procedures
}

Allen C, Glasziou P,Del Mar C. Bed rest: a potentially harmful treatment needing more careful evaluation. Lancet 1999

Oct 9;354:1229-33.

QUESTION: Is bed rest as effective as early mobilisation for patients with medical conditions or after healthcare procedures?

\section{Data sources}

Randomised controlled trials were identified by searching Medline and the Cochrane Library (1966 to June 1998) using terms related to bed rest (bed rest, bedrest, recumben [with various endings], expectant, and rest) and its opposite (early ambulat [with various endings], early activation, and early discharge). Personal files and bibliographies of relevant studies and review articles were also checked.

\section{Study selection}

Studies were selected if groups were studied in the same environment, treatments were the same except for the amount of bed rest prescribed, and treatments included drugs, surgery, or physical therapy but not education or advice.

\section{Data extraction}

Data were extracted on procedures (lumbar puncture, spinal anaesthesia, radiculography, cardiac catheterisation, skin graft for burns, liver biopsy, fixation of femoral fracture, pressure sore surgery, and ileus after gastric surgery) and conditions (acute low back pain, spontaneous labour, pregnancy induced hypertension with proteinuria, early threatened abortion, uncomplicated myocardial infarction, pulmonary tuberculosis, rheumatoid arthritis, and acute infectious hepatitis), ambulatory and bed rest procedures, and outcomes.

\section{Main results}

39 trials of 15 disorders (5777 patients) were included. Data were not pooled because of study diversity. 24 trials evaluated bed rest after medical procedures; only 8 outcomes differed and all showed worse outcomes for bed rest: nausea after lumbar puncture; severe headache after urological spinal anaesthesia; headache after obstetrical spinal anaesthesia; dizziness after radiculography of the lumbar spine; and haematoma, back pain or leg stiffness, pain, and back pain after cardiac comes favoured bed rest and 17 favoured early mobilisation.

15 trials evaluated bed rest as a primary treatment; only 8 outcomes differed and all showed worse outcomes for bed rest: disability at day 1 for acute low back pain; length of first stage of labour, contraction strength, assisted delivery, need for analgesia, and Apgar score at 5 minutes; venous thrombosis after myocardial infarction; and time for recovery after acute infectious hepatitis. Non-significant trends showed 6 outcomes favoured bed rest and 16 favoured early mobilisation. catheterisation. Non-significant trends showed 7 out-

\section{Conclusion}

Bed rest for primary treatment of medical conditions or after healthcare procedures is associated with worse outcomes than early mobilisation.

\section{COMMENTARY}

This systematic review by Allen $e t a l$ is valuable because it reminds clinicians that we often continue practices that have been shown to be ineffective. To illustrate this, the authors cite evidence that bed rest is still overprescribed after spinal puncture, myocardial infarction, cardiac catheterisation, and for acute low back pain despite studies that have shown its detrimental effects. On the basis of their review, Allen et al conclude that there is little support for bed rest in a wide range of settings and that it may delay recovery or harm the patient.

Studies selected for inclusion in the systematic review involved hospital and community settings. The authors specified tight inclusion criteria and appropriately did not pool study results because of the large variation among studies. Additional relevant studies may have been found if they had searched other databases such as CINAHL and if they had included unpublished studies. A wide range of conditions and procedures were studied, although many studies had small numbers of participants. It would be useful to know the age and ethnicity of the people studied, especially those requiring cardiac care and surgical interventions, because the complications of bed rest for older adults are more serious. ${ }^{1}$

The review will be a catalyst to many disciplines to re-evaluate their bed rest practice. The authors explain that clinicians must distinguish the use of bed rest in the management of symptoms, such as weakness, from its use as a prescribed treatment to speed recovery and should suggest that perhaps the patient is the best judge of the amount of rest required. The patient's level of activity is affected by the negotiation of care between nurses and patients. To be successful in encouraging patients to mobilise, nurses must find out what their patients understand about the purpose of activity. Patients, like some health professionals, may still think bed rest is necessary for healing, based on personal preferences or cultural beliefs.

Tish Butson, RN, MHSc Clinical Nurse Specialist, Gerontology Hamilton Health Sciences Corporation Hamilton, Ontario, Canada

Graduate School of

Medicine, University of

Queensland, Hersto

Queensland 4006,

Australia.Fax +617

33655442 .

A modified version of this abstract appears in ACP Journal Club. 